\title{
A STUDY ON PORT DESIGN AUTOMATION CONCEPT
}

\author{
Loo Hay Lee \\ Ek Peng Chew \\ Hai Xing Cheng \\ Yong Bin Han \\ Department of Industrial and Systems Engineering \\ 10 Kent Ridge Crescent, National University of Singapore \\ 119260, SINGAPORE
}

\begin{abstract}
In this paper, an automation concept is proposed to facilitate the simulation model building for port design problem. Currently, this process, which includes drawing the terminal layout and programming the simulation logic based on the drawn layout, is highly manual, very tedious and time-consuming. This makes the optimization of the port design very difficult because it involves too much manual process. Hence, we build an ALG (Automated Layout Generation) program to generate the simulation model automatically based on the input parameters provided by users. Besides, we integrate this program with simulation optimization algorithms, which can generate new designs, evaluate the designs efficiently and finally identify the promising designs.
\end{abstract}

\section{INTRODUCTION}

In the past decades, container throughput has been growing rapidly and vessels have also been increasing in size. Moreover, competition among ports is getting more aggressive. Hence, when designing new ports, we not only need to consider layouts and state-of-the-art equipment, but also need to identify new operation strategy which can exploit the equipment to achieve higher efficiency. The current way of designing a new port is usually based on past experiences. Port operators will come out with some design alternatives, and then built simulation models to evaluate and select the most favorable design. Due to the fact that developing simulation model requires highly manual and tedious processes, it is unlikely that many alternatives can be considered.

To build a simulation model, (without loss of generality, we use AutoMod for illustration) first we need to draw the port layout manually, which includes drawing the path for the vehicles, adding the control points for vehicles control and loads handling, and defining the work list and park list to control the vehicle's behavior. After finishing the layout drawing, we need to program the simulation logic to control the loads' behavior. The logic code is highly dependent on the layout drawn and the naming of the path, control points and resources. As a result, even when the layout is changed incrementally, the logic code needs to be rewritten substantially. The whole process to build a simulation model is very tedious and time-consuming since they are all done manually. This also makes it impossible to compare many layouts and hence may not find the best layout. To address the above issue, we build an ALG program to facilitate the generation of the layout and logic code for the simulation model. The ALG program will generate the necessary files which the simulation software can use to generate simulation models with different layout and logics based on the input from users. In addition, we integrate this ALG program with simulation optimization algorithms which is able to generate new designs, efficiently allocate simulation runs to different designs and identify promising designs.

The rest of this paper is organized as follows. Section 2 summarizes the related studies. Problem definition is presented in Section 3. In Section 4, the framework of the development of the system is given. Finally we give conclusions and future research directions.

\section{LITERATURE REVIEW}

Simulation is a powerful tool and is often used to evaluate alternative designs and explore possibilities. For example, Kozan (1997) conducted a comparison between the analytical and simulation planning models for a terminal. Bruzzone, Giribone, and Revetria (1999) showed the advantage and effectiveness of simulation approach for complex container port management. Yun and Choi (1999) also developed an object-oriented simulation model using SIMPLE++ to analyze the typical container terminal system in Pusan, Korea. Nam, Kwak, and Yu 
(2002) used simulation to examine the optimal number of berths and quay cranes to be used for a terminal in Busan, Korea. Shabayek and Yeung (2002) used simulation model to predict real operations for a terminal in Hong Kong. A simulation model was developed by Sgouridis and Yeung (2003) to study the inbound container handling in an "All-Straddle-Carrier" System. In Yang, Choi, and $\mathrm{Ha}$ (2004), a simulation model was developed to ana- lyze the effect of increasing the number of Automated Lifting Vehicles rather than Automated Guided Vehicles on the productivity of the terminals.

However, simulation model building usually is very time-consuming and tedious, which highly decreases simulation's power in decision making. In this paper, we will develop the ALG program to automate the process of simulation model building.

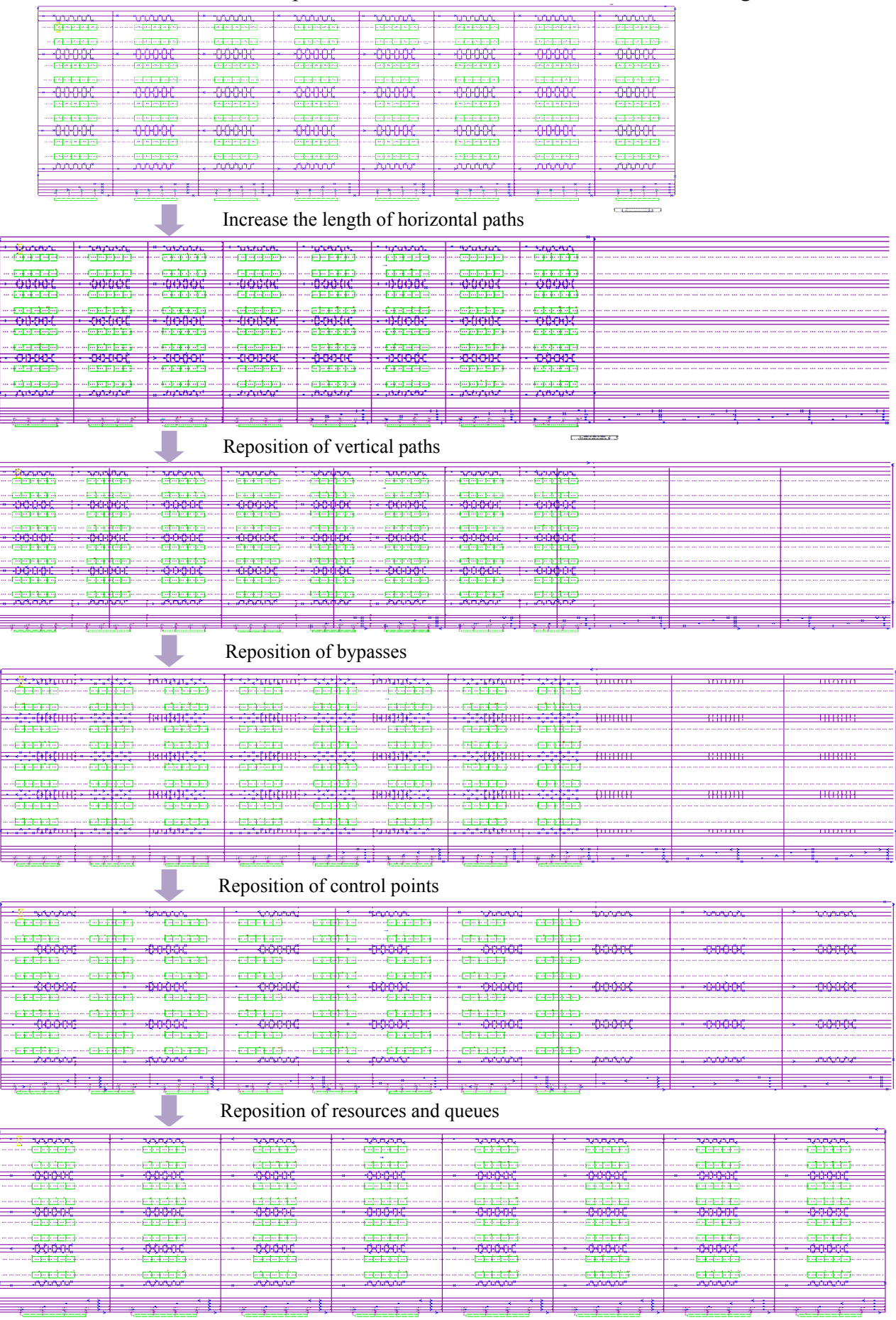

Figure 1: Manual process of changing layout. 


\section{PROBLEM DEFINITION}

This study is motivated by the actual port layout design process using simulation software. In most of the time, it is very time-consuming to draw a detailed layout and build a complete simulation model by any simulation software. After the simulation model is built, when port layout designers want to fine-tune the original layout design, or make some minor modifications on the given layout, they always need to rebuild the simulation model because most of the simulation software does not have the flexibility to automatically change the simulation model when some of the layout specifications are changed. For example, when users want to increase the length of horizontal paths, they will also need to manually change the vertical paths, edit control points on the paths and the fillet paths, and reposition the resources and queues one by one (see Figure 1).

When users attempt to change more than one design parameters at the same time, for example, change the total length and width of the layout together, the time and effort needed will be even more. Hence, it will be computationally infeasible if users want to try a lot of different designs. This difficulty has highly decreased the usefulness of simulation in layout design optimization, and made layout design optimization become very difficult.

The scope of this study is to verify the feasibility of the automation concept, and provide a prototype for the ALG program and the integrated simulation optimization framework. The ALG program will be developed based on a port with horizontal layout. The simulation optimization framework will integrate the ALG program with the search algorithm and the optimal computing budget allocation algorithm (OCBA) to search for the promising design according to a single performance indicator which in this case will be the average vessel turnaround time.

\section{DEVELOPMENT OF SYSTEM}

\subsection{Development of the ALG Program}

The purpose of ALG program is to automatically generate the simulation model based on input provided by users. It is coded based on a layout template and the port operation logic. The layout template defines the relative position of the paths and resources, while the port operation logic defines the rules on how containers are transported, how the equipments handle containers, the queuing disciplines, ..etc.. For this prototype model we illustrate with a specific port design, i.e., the port with a horizontal layout, which can be commonly found it Asia.
First we store all the design parameters in a ".csv" input file (Microsoft Office Excel Comma Separated Values File). The ALG program will read the input information from this file, and then generates several AutoMod model files, which includes pm.asy, yc.asy, layout .asy. The first two files are for creating the path mover systems for prime movers and yard crane respectively while the layout .asy is for creating the process system which includes the simulation logic and resources definition, etc. With these files, we can run the simulation with Automod. When we want to consider different designs, we only need to specify the information in the .csv file (see Figure 2). A sample of the run for the ALG program is shown in Figure 3.

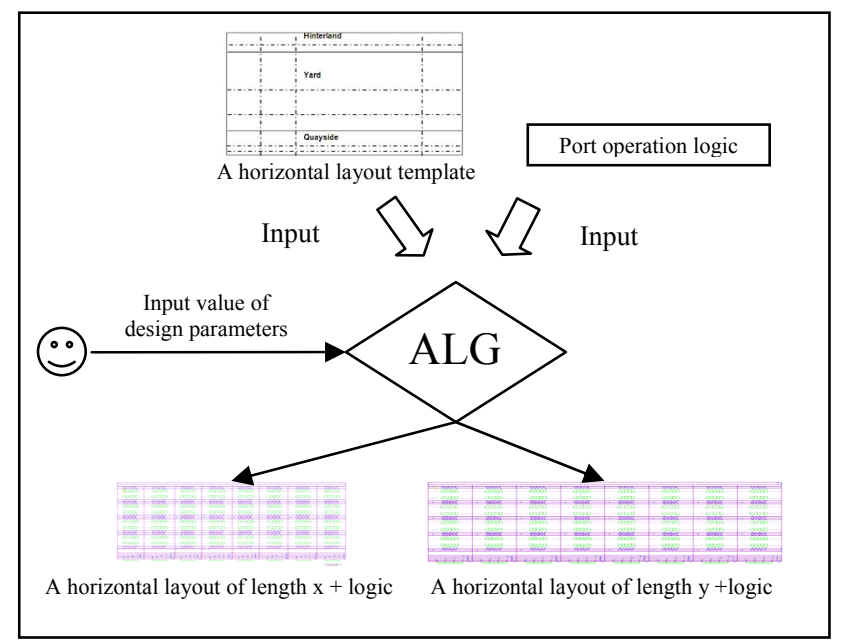

Figure 2: Framework for the ALG program.

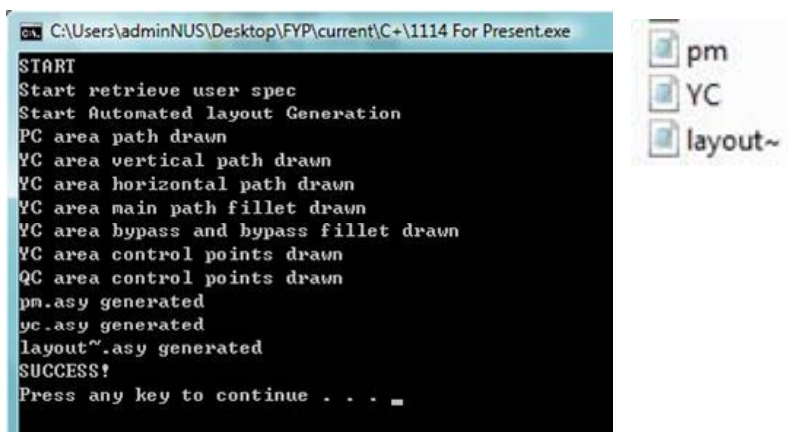

Figure 3: Program running screen shot.

With the ALG program, the process of changing some design parameters and creating new simulation model become far more efficient than before. A new model will be created in a few seconds after changing the input scv file and running the ALG program. Below are some illustrations: 
- Change one parameter in the original layout (see Figure 4) by increasing the length of the layout by $50 \%$. The result is shown in Figure 5.

- Change few parameters at the same time in the original layout e.g., decrease the length of the layout, increase the width of the layout, increase the number of blocks vertically, and decrease the number of blocks horizontally. The result is shown in Figure 6.

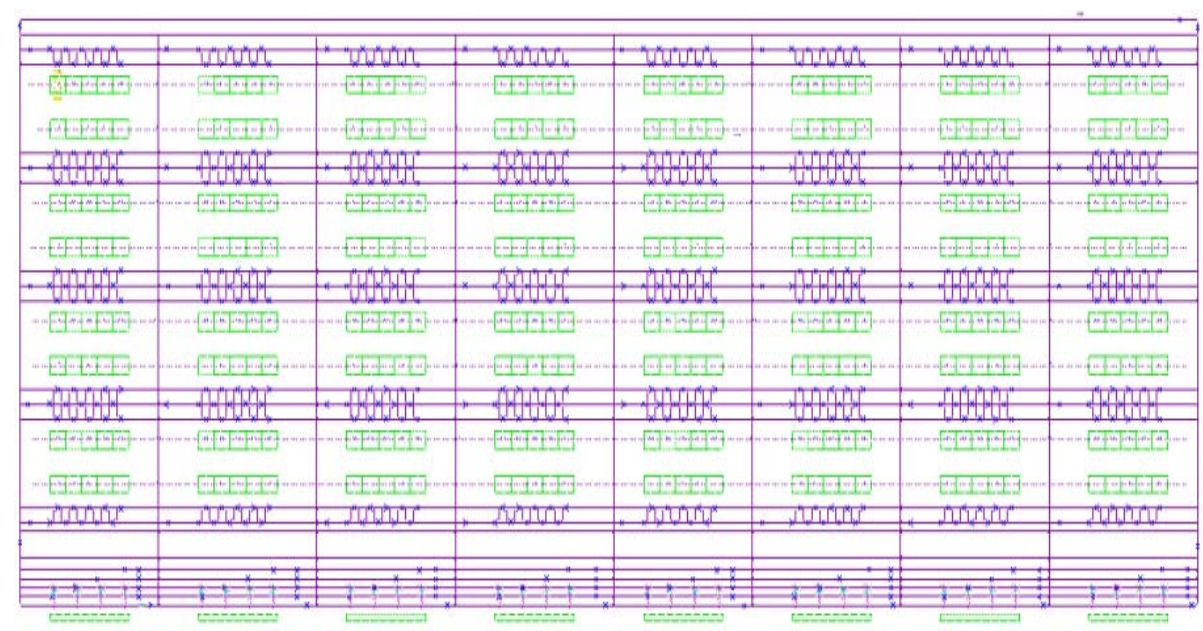

Figure 4: The original yard layout.

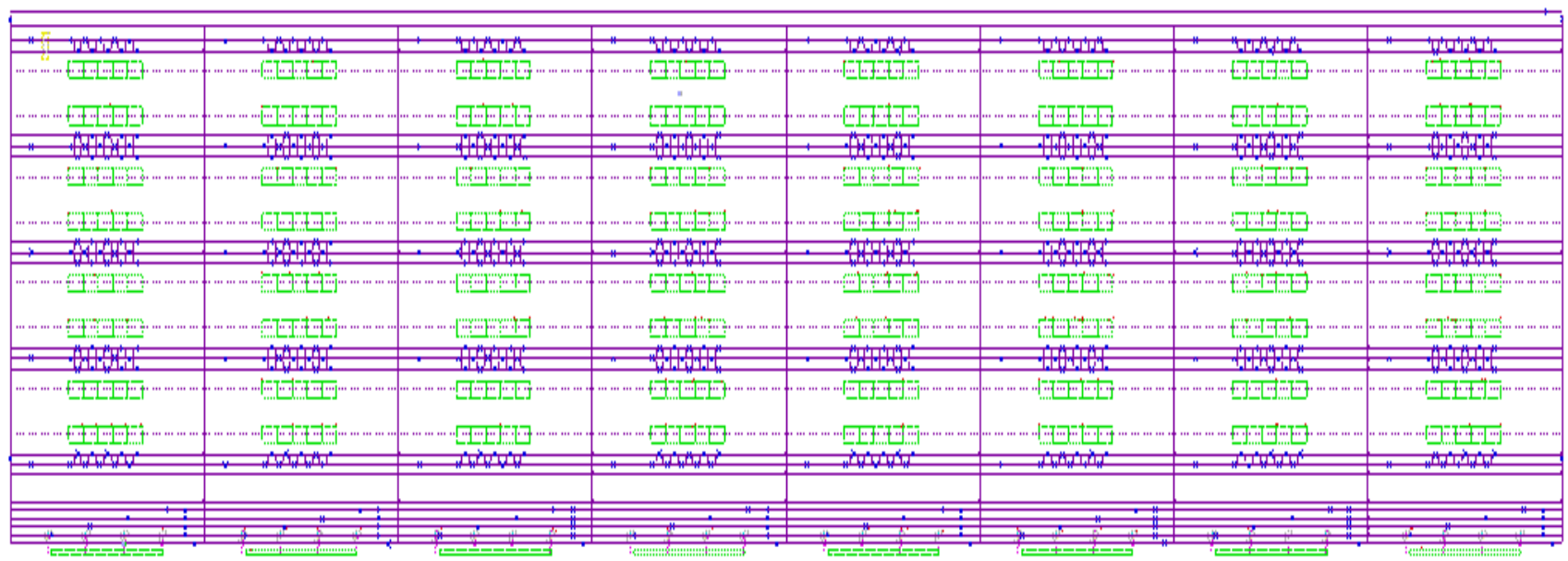

Figure 5: The updated layout with single-parameter change. 


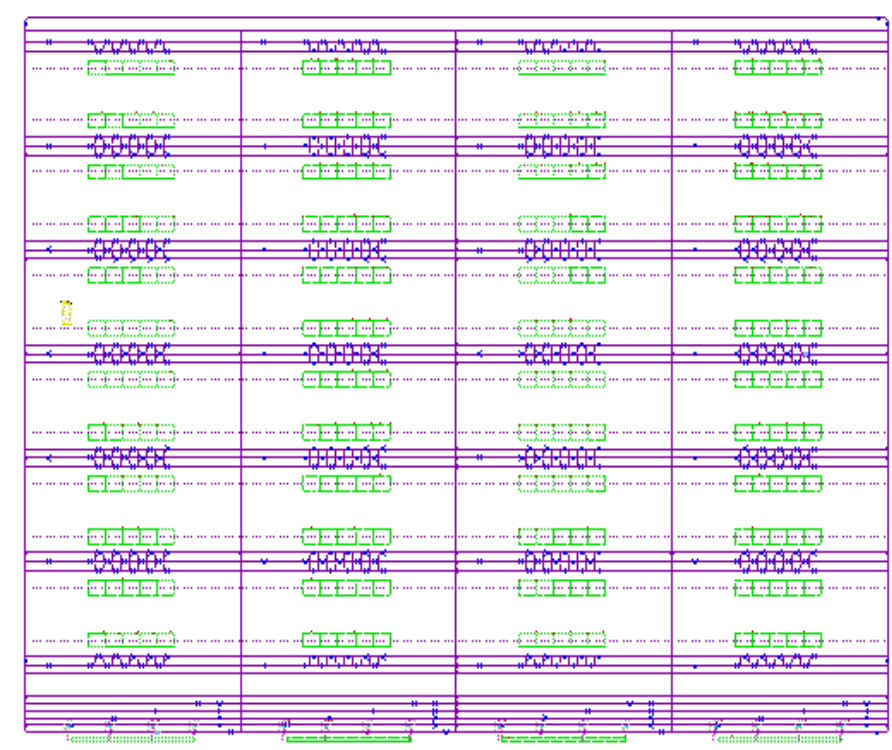

Figure 6: The updated layout with multi-parameter changes.

\subsection{Development of Integrated Framework for Port Layout Design}

By leveraging the ALG's automation power, the second objective of the study is to develop an integrated simulation optimization framework for port design problem. The framework integrates the ALG program and some simulation optimization algorithm to search for promising designs (see Figure 7). It is especially useful when the searching space is large, where exhaustive search is impractical or computationally infeasible.

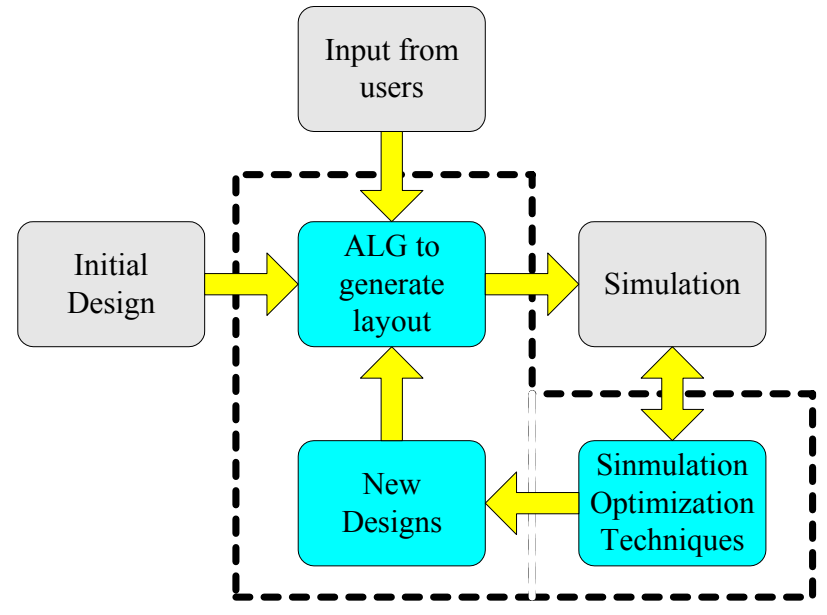

Figure 7: Flowchart for the integrated framework.
Similar to the input of the ALG program, the input for the integrated framework is in a .csv file. The difference is that for the design parameters, instead of a fixed value, a range is given.

The simulation optimization algorithm will first generate a few designs after reading the ".cvs" file. After that, ALG will be used to generate simulation models for these designs. Then optimal computing budget allocation algorithm (Chen et al. 2000, Lee et al. 2008) is used to determine the required simulation replications for each design based on the initial simulation results. After that, promising designs will be selected to generate new designs based on some search algorithms.

\section{CONCLUSION AND FUTURE RESEARCH}

\subsection{Conclusion}

In this paper, we present an automated layout generation concept and a simulation optimization framework for port design problem, which aims to facilitate the simulation model building process. With the implementation of this framework, the power of simulation can be utilized further in port design.

The ALG program is built to generate the files needed by the simulation software. To leverage on the power of the ALG program, we further integrate it with some simulation optimization algorithms to search for promising designs. 
The successful realization of these concept gives potential for further research in this field.

\subsection{Future Directions}

The next stage of research is to expand the scope and the content for the program, so as to increase its application to a wider range of problems. The work includes:

- Creating a library of layout templates, which includes both horizontal and vertical layout design;

- Creating a library of port operation logics, like different vessel berth allocation, quay crane assignment and yard storage location allocation rules;

- Providing a more complete list of design parameters;

- Using a more advanced searching engine, e.g. Genetic algorithm (GA), or other meta heuristics;

- Extending single performance to multiperformance.

\section{REFERENCES}

Bruzzone, A. G., P. Giribone, and R. Revetria. 1999. Operative requirements and advances for the new generation simulators in multimodal container terminals. In Proceedings of the 1999 Winter Simulation Conference, 1243-1252, Phoenix, Arizona, United States.

Chen, C. H., J. W. Lin, E. Yücesan, and S. E. Chick. 2000. Simulation budget allocation for further enhancing the efficiency of ordinal optimization. Discrete Event Dynamic Systems: Theory and Applications 10: 251-270.

Lee, L. H., E. K. Chew, S. Y. Teng, and Y. K. Chen. 2008. Multi-objective simulation-based evolutionary algorithm for an aircraft spare parts allocation problem. European Journal of Operational Research 189(2): 476-491.

Nam, K. C., K. S. Kwak, and M. S. Yu. 2002. Simulation study of container terminal performance. Journal of Waterway, Port, Coastal and Ocean Engineering 128(3): 126-132

Kozan, E. 1997. Comparison of analytical and simulation planning models of seaport container terminals. Transportation Planning and Technology 20(3):235248.

Sgouridis, S. P., D. Makris, and D. C. Angelides. 2003. Simulation analysis for midterm yard planning in container terminal. Journal of Waterway, Port, Coastal and Ocean Engineering 129(4):178-187.

Shabayek, A. A., and W. W. Yeung. 2002. A simulation model for the Kwai Chung container terminals in Hong Kong. European Journal of Operational Research 140:1-11.
Yang, C. H., Y. S. Choi, and T. Y. Ha. 2004. Simulationbased performance evaluation of transport vehicles at automated container terminals. OR Spectrum 26:149170.

Yun, W. Y., and Y. S. Choi. 1999. A simulation model for container-terminal operation analysis using an object-oriented approach. International journal of Production Economics 59(1-3):221-230.

\section{AUTHOR BIOGRAPHIES}

LOO HAY LEE is an associate professor in the department of Industrial and Systems Engineering at National University of Singapore. His research interest lies in the area of production planning and control, logistics and vehicle routing, supply chain modeling, simulation-based optimization, ordinary optimization, and evolutionary computation. His web page can be found via <http://www.ise.nus.edu.sg/staff/leelh /index.html>.

EK PENG CHEW is an associate professor in the department of Industrial and Systems Engineering at National University of Singapore. His research interests include logistics and inventory management, system modeling and optimization, analysis of order picking systems, and optimization. His web page can be found via <http://www.ise.nus.edu.sg/staff/chewe $\mathrm{p} /$ index.html $>$.

HAI XING CHENG is an undergraduate student in the department of Industrial and Systems Engineering at National University of Singapore.

YONG BIN HAN is a research fellow in the department of Industrial and Systems Engineering at National University of Singapore. His research interest lies in the area of simulation and optimization in port operations. 